\title{
The Effect of Coloured Overlays on Reading Ability in Children with Autism
}

\author{
Amanda K. Ludlow, ${ }^{1,3}$ Arnold J. Wilkins, ${ }^{2}$ and Pam Heaton ${ }^{1}$
}

\begin{abstract}
Abnormalities of colour perception in children with autistic spectrum disorders have been widely reported anecdotally. However, there is little empirical data linking difficulties in colour perception with academic achievement. The Wilkins Rate of Reading Test was administered with and without Intuitive Coloured Overlays to 19 children with autistic spectrum disorders and to the same number of controls individually matched for age and intelligence. Findings showed that 15 out of 19 (79\%) children with autism showed an improvement of at least $5 \%$ in reading speed when using a coloured overlay. In contrast only 3 of 19 (16\%) control group children showed such an improvement. The findings suggest that coloured overlays may provide a useful support for reading for children with autism.
\end{abstract}

KEY WORDS: Autism; colour perception; reading; academic achievement; coloured overlays.

Although autism is characterised by deficits in socialisation, communication and imagination (Diagnostic and Statistical Manual of Mental Disorders: DSM-1V, 1994), this paper will focus on dysfunction in perceptual information processing. Kientz \& Dunn (1997) report that children with autism show sensitivities to visual and other sensory stimuli in everyday life. However, there have been few well-controlled studies investigating hypersensitivity of this kind, and tests of sensory function have shown that such abnormalities are difficult to quantify (Cummins, Heaton, \& Happe: in preparation). Methodological shortcomings such as a failure to control for age and intelligence, mean that findings from studies can be

\footnotetext{
${ }^{1}$ Department of Psychology, Goldsmiths College, University of London, New Cross, London, UK.

${ }^{2}$ Department of Psychology, Essex University, Wivenhoe Park, CO4 3SQ Colchester, UK.

${ }^{3}$ Correspondence should be addressed to: Amanda K. Ludlow, Department of Psychology, Goldsmiths College, University of London, New Cross, SE14 6NW London, UK.; e-mail: ps901akl @ gold.ac.uk
}

difficult to interpret. Additionally, it is currently unclear whether or not sensory processing abnormalities are specific to autistic spectrum disorders or are the result of general intellectual impairment.

Idiosyncratic responses to colours have been widely reported in autism. For example, parents have reported that their autistic children refuse to eat green food, will only drink from a red cup or always choose blue items to the exclusion of most others. One adult with autism reported how as a child he refused to look at the yellow bike given to him for Christmas because of its colour. He recalled how, when extra red was added to the bike, it became orange and looked as though it was on fire (White \& White, 1987).

Howlin (1996) has reported that some individuals with autism improve in physical and emotional functioning as well as in intellectual capacity after lengthy observation of coloured lights. Syntonics, or Optometric Phototherapy is an ocular science that treats a wide range of visual problems by applying visible light frequencies to the eyes. Unfortunately, no rigorous studies into the therapeutic benefits of 
such treatments have been carried out (Evans \& Drasdo, 1991) and how such applications might benefit autism is yet to be investigated.

Williams (1999) "a high functioning woman" with autism has reported how different coloured light bulbs influence her mood and the degree of comfort and accuracy with which she can see things. She claims "The red had me alert and aware and I started to look for things to do within the room instead of staring hypnotically at the mirror or wallpaper" (Williams, 1999, p. 140). Given these reports, it is surprising how little research into colour perception in autism has been carried out.

Irlen (1991) reports that one cognitive ability that has been shown to be influenced by colour processing is reading. Reading can provoke pattern glare that causes eye-strain, perceptual distortions such as blurring or apparent movement of letters, and illusions of shapes or colours on the page (Wilkins \& Nimmo-Smith, 1987; Wilkins et al., 1984). The term Meares-Irlen syndrome (also known as Scotopic Sensitivity Syndrome or Irlen syndrome) refers to the signs and symptoms of visual stress that are associated with discomfort when reading. Individuals with Meares-Irlen syndrome often report that the perceptual distortions they experience when they read are reduced when text is illuminated by light of a particular optimal colour (Evans, Wilkins, Busby, \& Jeanes, 1996b; Wilkins, Jeanes, Pumfrey, \& Laskier, 1996). When the individuals wear spectacles tinted with this colour, they have reduced symptoms such as eye-strain and headaches (Wilkins et al., 1996). Other studies have shown that coloured overlays eliminate symptoms and increase reading speed in these individuals (Irlen, 1991; Jeanes et al., 1997; Wilkins, 2003; Wilkins, Lewis, Smith, \& Rowland, 2001).

Colour overlays are transparent coloured plastic sheets that can be placed over printed texts without interfering with their clarity. Participants self-select the overlay that they perceive best improves the clarity of the printed text. The chosen overlay can be any one from the two sets of identical 10 (9 coloured and 1 grey) provided in the Intuitive Overlays Selection pack (Wilkins et al., 1994). Participants can use a single overlay or they can use stronger colours in the form of double overlays. Research using this test has been carried out with children in mainstream schools and has shown that $5 \%$ of children aged 7-11 read more than $25 \%$ faster and $20 \%$ read more than $5 \%$ faster with a coloured overlay (Wilkins et al., 2001). In addition to improvement in reading speed with coloured overlays, corresponding improvements in reading accuracy have been found (Wilkins et al., 1996). Researchers hypothesise that the rate of improvement in reading speed and accuracy in the adult population is similar to that of children (Evans \& Joseph, 2002).

Although the Intuitive Overlays allow freedom of selection to participants, the colour choice tends to be consistent over testing sessions. For example, on two separate testing sessions, three days apart, 78 children (87\% of those in the study) chose to read a text with a coloured overlay rather than without. Of these, $47 \%$ chose the same colour and $21 \%$ chose an overlay of a similar colour (neighbouring chromaticity). Children who chose exactly the same colour on both occasions showed the greatest improvement in reading (Wilkins et al., 2001). In another study, 368 children were given a random rather than selfselected overlay in the classroom. Those who were given their chosen overlay or one that was of similar colour elected to use the overlays for a longer period than the children who had been given random ones (Wilkins et al., 2001).

Studies carried out with typically developing children have shown that the benefit of overlays is not simply due to placebo effects. One such research design, adopted in two separate studies (Bouldoukian, Wilkins, \& Evans, 2002; Wilkins \& Lewis, 1999) compared reading rate with no overlay, with a chosen overlay, with a grey overlay, and a grey overlay that was identical except that it was labelled, "scientific prototype". Children were told that the prototype was new and combined all the colours and that they would be the first to use it. However, the findings from the study showed that improvements in reading were only seen in those who had chosen a coloured overlay and no placebo effects were found. In another study the rate of reading test was carried out in five different conditions: without an overlay, with a clear (transparent) overlay, with the grey overlay from the intuitive overlays, and with two coloured overlays from the same set, one of the chosen colour and one of a colour opposite (complementary) to that chosen. Again rate of reading was only superior in the chosen colour overlay condition (Jeanes et al., 1997).

It would seem logical that the proportion of individuals who benefit from overlays will be overrepresented in populations of people with reading difficulties such as dyslexia. This was originally proposed by Irlen (1991). Dyslexia is evident when accurate and fluent word reading and/or spelling develops incompletely or with great difficulty (British Psychological Society, 1999) and it is the most 
common of the learning difficulties. Some of the subtle visual deficits seen in individuals with dyslexia are similar to those found in people with MearesIrlen syndrome (Evans, 2001). However, recent findings have shown that individuals with dyslexia benefit from overlays only slightly more frequently than the rest of population (Kriss, 2002). Many of the symptoms of Meares-Irlen syndrome are not specific to this disorder. For example, children with attention deficit/hyperactivity disorder (ADD/ADHD) share symptoms with both Meares-Irlen syndrome and dyslexia, and these include difficulties in tracking words and lines when reading, and poor concentration (Stone, 2002). Thus whilst overlay use has not yet been shown to benefit any one specific group, children with reading difficulties are more likely than others to report visual perceptual distortions. As coloured overlay use appears to be one way of removing these distortions they may be especially beneficial to these children with reading difficulties.

Williams (1999) "an able adult with autism" was one of the first individuals to report the therapeutic effects of coloured filters. She describes hyper-acute vision that results in a tendency to focus on minute details and reports that tinted lenses enable her to view the world clearly and wholistically. She has proposed that many individuals with autism would also benefit from coloured lenses.

Although the mechanisms of benefit are currently uncertain, Wilkins (2003) has presented evidence that peripheral mechanisms are insufficient to explain the phenomenon, and that the mechanisms are cortical in origin. Children who benefit from the overlays are twice as likely to come from families with a history of migraine than those who do not benefit (Maclachlan, Yale, \& Wilkins, 1993) and in individuals with migraine the cortex is hyperexcitable (Aurora \& Welch, 1998; Huang, Cooper, Satana, Kaufman, \& Cao, 2003). Visual stimuli that provoke photophobia, a negative reaction to bright light, are similar to those responsible for causing seizures in patients with photosensitive epilepsy (Wilkins, 1995). Awareness of the distortions can occur in the normal population, although people with migraine are particularly susceptible (Chronicle \& Wilkins, 1991; Chronicle, Wilkins, \& Coleston, 1995; Marcus \& Soso, 1989). Wilkins (2003) proposes that the distortions are the result of a spread of activation within the cortex that causes cells to fire inappropriately. The spread of activation is a reflection of cortical hyperexcitability, similar to that in epilepsy, though less extreme. In photosensitive epilepsy the hyperexcitability can be diffuse, but is not necessarily uniform, and sometimes appears to involve only a few cortical orientation columns (Wilkins, 1995). Xiao, Wang, \& Fellerman (2003) have shown that in visual area V2 colour sensitive cells are distributed topographically according to chromaticity and Wilkins (2003) has proposed that appropriately coloured filters change the distribution of firing within the cortex so as to reduce the excitation in hyperexcitable regions. Findings from fMRI studies investigating the hyperexcitability that occurs in migraine (Huang et al., 2003; Huang, Wilkins, \& Cao, 2004) have provided preliminary support for this suggestion. The cortical hyperexcitability hypothesis predicts that coloured filters should be of benefit in a wide range of central nervous system disorders in which the visual cortex is hyperexcitable. In support of this are studies showing that coloured glasses are beneficial in photosensitive epilepsy (Wilkins \& Lewis, 1999) migraine (Evans \& Joseph, 2002; Wilkins, Patel, Adjamian, \& Evans, 2002) and head injury (Jackowski, Sturr, Taub, \& Turk, 1996).

Individuals with autism are liable to epileptic seizures (Bryson, Clark, \& Smith, 1988; Cialdella \& Mamelle, 1989; Deykin \& MacMahon, 1979; Ornitz, 1973; Rutter, 1970; Steffenburg \& Gillberg, 1986; Tanoue, Oda, \& Kawastima, 1988; Wing \& Gould, 1979) and for one third of this population epileptic seizures are experienced from early in adult life (Gillberg, 1991). It is therefore possible that some of the perceptual distortions they report reflect cortical hyperexcitability. If so, it is also possible that coloured filters may alleviate the distortions and exert beneficial effects, for example, on reading ability.

Although the majority of children with autism also have a degree of intellectual impairment, many learn to read (Bartak \& Rutter, 1975; Cobrinik, 1974; Lockyer \& Rutter, 1969). Therefore it is possible to investigate changes in reading with and without coloured overlays. The present study will test the hypothesis that a greater proportion of children with autism will read faster and more accurately with overlays than controls matched for verbal IQ and chronological age.

\section{METHOD}

\section{Participants}

Nineteen children with autism participated in the experiment. These children were aged between 8 years and 4 months and 15 years and 1 month 
(mean 11 years and 10 months). All children attended schools for which a formal diagnosis of autism was the criterion for entry. Their scores on the British Picture Vocabulary Scale (BPVS) (Dunn, Dunn, Whetton, \& Burley, 1997) ranged between 47 and 87 (mean 64.3). Control participants were typically developing children recruited from mainstream schools and children with moderate learning difficulties recruited from special needs schools. These children were matched to the children with autism on an individual basis for chronological age, gender and verbal IQ as measured by the British Picture Vocabulary Scales (Dunn et al., 1997). All children also completed two colour abnormality tests: the Ishihara Test (Ishihara, 1970) and the City University Colour Vision Test (3rd edn; Fletcher, 1998). The Ishihara Test provides a sensitive measure of the red/green confusion associated with protanomly and deuteronomaly. The City University Colour Vision Test (3rd edition; Fletcher, 1998) involves discrimination of shades of colour and includes a measure for tritan defects which can cause difficulty with the colour blue and some other colours. These data, together with age and verbal IQ data are shown in Table I below.

\section{Materials}

The Intuitive Overlays are sheets of coloured plastic sheet suitable for placing over a page of text so as to colour the text beneath without interfering with its clarity. They sample chromaticity systematically and efficiently (Wilkins, 1994). They are supplied in a teacher's pack and include two A5 size overlays of each of the following colours: rose, orange, yellow, lime green, mint green, aqua, blue, purple, pink and grey.

The Rate of Reading Test is a passage consisting of 20 lines, each with the same 15 common words in a different random order. The words are of high frequency and therefore familiar to poor readers. The random word order ensures that no word can be guessed from the context but each must be seen to be read. Absence of meaning has the advantage that children are often unaware of the errors of omission and insertion of words. The passage is read out loud for a minute and the score is the number of words read correctly in the appropriate order (Wilkins et al., 1996). The published test exists in two versions that differ only as regards typeface, size and spacing. The larger text (14 pt Geneva) was used.

\section{Procedure}

A test page consisting of two A4 pages with two identical passages of the Rate of Reading text side by side was positioned in front of the child at a reading distance of $0.4 \mathrm{~m}$. The page was positioned so that no light sources were directly reflected from the surface of the overlays. Children were asked to read the passage for 30 seconds and told that it was a practice run and that the passage did not make sense. Then while the children were still looking at the passage they were asked the following visual stress questions - "Do the letters stay still or do they move?"; "Are they clear or are they blurred (fuzzy, difficult to see)?"; "Are the words too close together or far enough apart?"; "Is the page too bright, not bright enough, or just about right"; "Does the page hurt your eyes to look at or is it ok?". The questions reveal symptoms that are generally slightly greater in those who show improvements in reading speed with overlays (Wilkins et al., 2001). All of the participants in the study were verbal, and gave ready responses to the questions asked. No children asked for clarification on any of the questions asked. A score of 1 was given for each visual stress symptom expressed (these are underlined), others responses scored 0 .

The colour overlays were assembled in a pile in the following order: Rose, Lime-Green, Blue, Pink, Yellow, Aqua, Purple, Orange, Mint-Green. The order was designed to avoid complementary colours

Table I. Age, Verbal IQ and Colour Test Data for Children with Autism and Controls

\begin{tabular}{|c|c|c|c|c|c|c|c|c|}
\hline \multirow[b]{2}{*}{ Group } & \multicolumn{2}{|c|}{ Age } & \multicolumn{2}{|c|}{ BPVS } & \multicolumn{2}{|c|}{$\begin{array}{l}\text { City Univ. Colour } \\
\text { Test }(\max =16)\end{array}$} & \multicolumn{2}{|c|}{$\begin{array}{c}\text { Ishihara Colour } \\
\text { Test }(\max =38)\end{array}$} \\
\hline & Mean & $\mathrm{SD}$ & Mean & $\mathrm{SD}$ & Mean & SD & Mean & SD \\
\hline Children with autism $(n=19)$ & 11.10 & $(2.23)$ & 64.32 & $(10.91)$ & 15.9 & $(0.33)$ & 36.7 & $(1.04)$ \\
\hline Controls $(n=19)$ & 11.9 & $(1.95)$ & 68.36 & $(12.75)$ & 15.9 & $(0.34)$ & 35.7 & $(1.21)$ \\
\hline
\end{tabular}


being placed next to each other. The top overlay (Rose) was placed on the left hand side of the test page, covering one of the two passages of text, matt side uppermost. Children were asked which side was the clearest and most comfortable to see. If the white side (passage without overlay) was best then the overlay was removed and replaced with the next overlay from the pile. If the coloured overlay was selected as the best then the overlay was turned over to see whether the matt side or the gloss side was preferred. The preferred side was then used for the remaining overlays.

When the overlay was chosen as being preferable to the uncovered side, it was positioned with best side uppermost and another overlay was placed in the opposite side of the page so that both pages were now covered with overlays. The child was then again asked which side was the clearest and most comfortable. This process was continued each time leaving the best overlay in place and removing the poorer overlay, replacing it with the next from the pile. When two colours were deemed indistinguishable both colours were noted and one of the colours changed. The other colour was re-introduced at the end of the pile.

Finally, the researcher checked the child's preferred overlay compared to no overlay to be sure of the best final selection. When the children had selected an overlay, researchers asked questions about visual stress again.

Stronger colours in the form of double overlays were next used to see if they made the text clearer than a single overlay. Stronger colours of the chosen hue were obtained by placing two overlays of the same or neighbouring chromaticity on top of each other to form a double overlay, as described in the Intuitive Overlays instruction booklet. The best single overlay was compared with the three associated double overlays and the best chosen by a process of elimination. If a double overlay was preferable, the questions concerning symptoms were repeated a third time.

The chosen overlay or combination of two overlays was then placed over one of the two passages on the test page, covering the other passage with a mask. The children were asked to read the text aloud for 1 minute as quickly as possible and were timed using a stopwatch. The total number of words read in the correct order was noted. The overlay(s) were then removed and the children were asked to read the text again for a minute. The order in which the children received the overlay was randomised within groups so that some children read first with the overlay and others read first without the overlay.

\section{RESULTS}

\section{Coloured Overlays and Rate of Reading Test}

Three of the controls did not choose an overlay and preferred the text plain. These three children were not included in the analysis.

Previous studies using the Intuitive Colour Overlays have set a $5 \%$ increase in reading speed as the criteria for significant effects (Wilkins, 2003). Therefore an initial analysis, using a $2 \times 2$ chi-square was carried out. This showed that significantly more children with autism read faster at the 5\% level with the overlays, $\chi^{2}=12.600 ; \mathrm{df}=1, p<0.001$.

A $2 \times 2$ ANOVA was then carried out on the data. Group (autism/controls) was the between factor and condition (number of words read per minute with and without overlays) as the within group factor. The analysis showed no significant main effect of condition $(F(1,33)=1.261$, n.s. $)$ or group $(F(1,33)=1.913$, n.s.). However, there was a significant group $\times$ condition interaction $F(1,33)=15.475, p<0.05$. This is shown in Fig. 1 below.

This interaction was analysed using paired $t$-tests. These showed that children with autism read significantly more words per minute with than without colour overlays $(t(18)=-3.28, p<.004)$. The difference was also significant for controls although here performance was poorer with overlays $(t(15)=2.39, p<0.031)$. No significant between group difference (autism/controls) was found when children read without colour overlays $(t(33)=.516$, n.s.) but children with autism read significantly faster than controls with colour overlays $(t(33)=2.26, p<0.03)$.

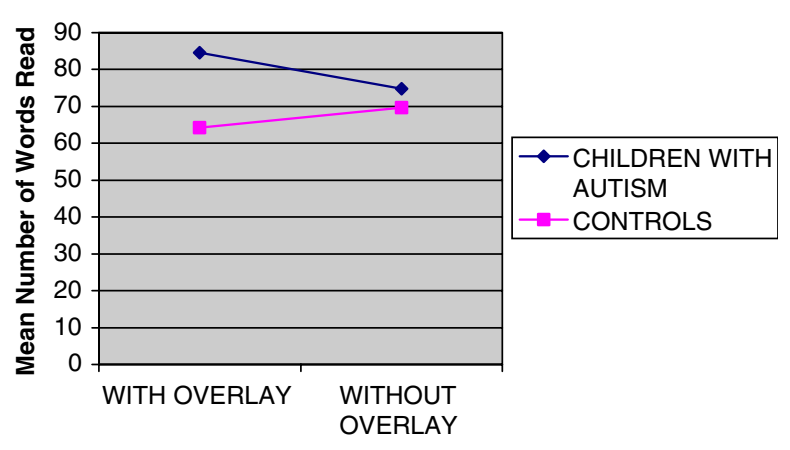

Fig. 1. Group by condition interaction. 
As there was a high degree of variability in scores, particularly for the children with autism, individual data are shown in Table II and Figs. 2 and 3 below.

\section{Visual Stress}

For those children (from both groups) who chose an overlay, an average of 1.17 (SD 1.043) symptoms of visual stress were reported without the overlay compared with 0.69 (SD 1.157) with the overlay. This difference is statistically significant, $t(34)=3.022$, $p<0.05$. For those children who showed greater improvements (more than 5\% faster) with an overlay, an average of 1.37 (SD 1.257) symptoms were reported without the overlay compared to 1.05 (SD 1.433). The difference was not statistically significant, $t(18)=1.189$, n.s.

In order to determine whether there was a relationship between verbal IQ, as measured by the BPVS and degree of improvement using overlays, a difference score (score with overlay minus score without overlay) was calculated and correlated with VIQ. There was no significant correlation $(r=-0.208$, n.s. $)$.

There was no marked overall preference for any specific colours within either group. The distribution of colour overlays chosen by individual children are included in Appendix A.

\section{DISCUSSION}

The present study is the first to investigate the influence of coloured overlays on the rate of reading in children with autism. The results from the study showed that when a $5 \%$ increase in reading speed was accepted as the criterion for significant improvement, more children from the autism group increased reading speed than children from the age- and intelligence-matched control group. However, inspection of individual data showed that many of the children with autism increased reading speed at higher levels. Thus whilst $79 \%$ of the children with autism read more than 5\% faster with overlays, $67 \%$ of these children increased reading speed at rates ranging between 11 and 50\% with overlays. The range of improvement in the small proportion of controls who did reach or exceed the 5\% improvement criterion was smaller, spanning 8-25\%. Research using colour overlays with normal populations has shown a $5 \%$ increase in $20 \%$ of individuals (Wilkins et al., 2001). These results are consistent with the findings from the control group in the current study in which $16 \%$ of individuals showed an increase of more than $5 \%$ with overlays. However the numbers of children within the autism group who improved in reading speed using colour overlays was significantly

Table II. The Percentage of Improvement in Reading in the Group with Autism and Their Controls with a Colour Overlay

\begin{tabular}{|c|c|c|c|c|c|}
\hline \multirow{2}{*}{$\begin{array}{l}\text { Numbers of children showing } \\
\text { improved reading with overlays }\end{array}$} & \multicolumn{5}{|c|}{ Percentage improvement reading with colour overlays } \\
\hline & $5-10 \%$ & $11-20 \%$ & $21-30 \%$ & $31-40 \%$ & $41-50 \%$ \\
\hline Autism 15 (out of 19 ) & $3(16 \%)$ & $6(31 \%)$ & 0 & $4(21 \%)$ & $2(10 \%)$ \\
\hline Controls 3 (out of 19 ) & $1(5 \%)$ & $1(5 \%)$ & $1(5 \%)$ & 0 & 0 \\
\hline
\end{tabular}

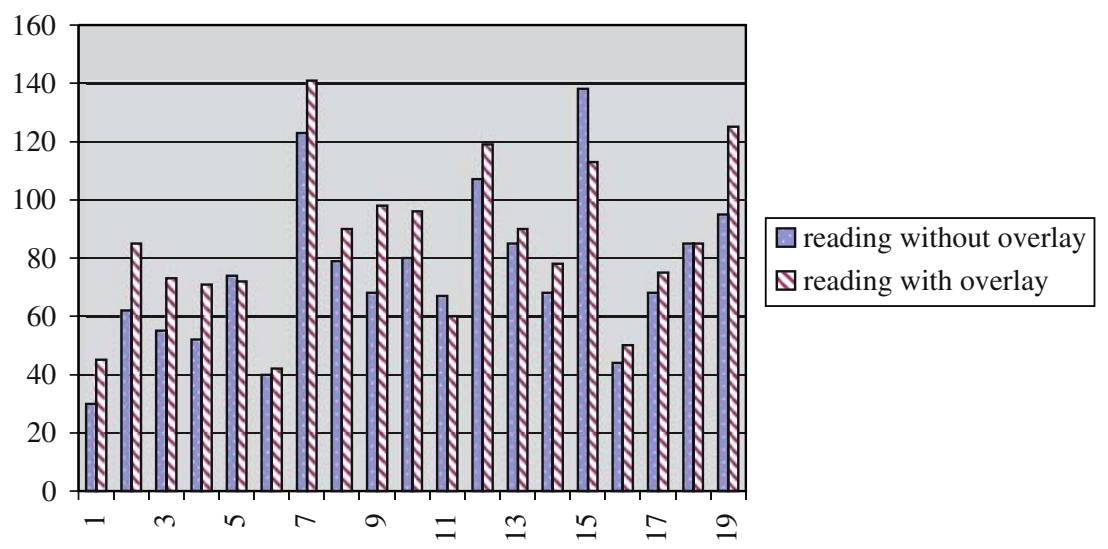

Fig. 2. Words read per minute with and without colour overlays for children with autism. 


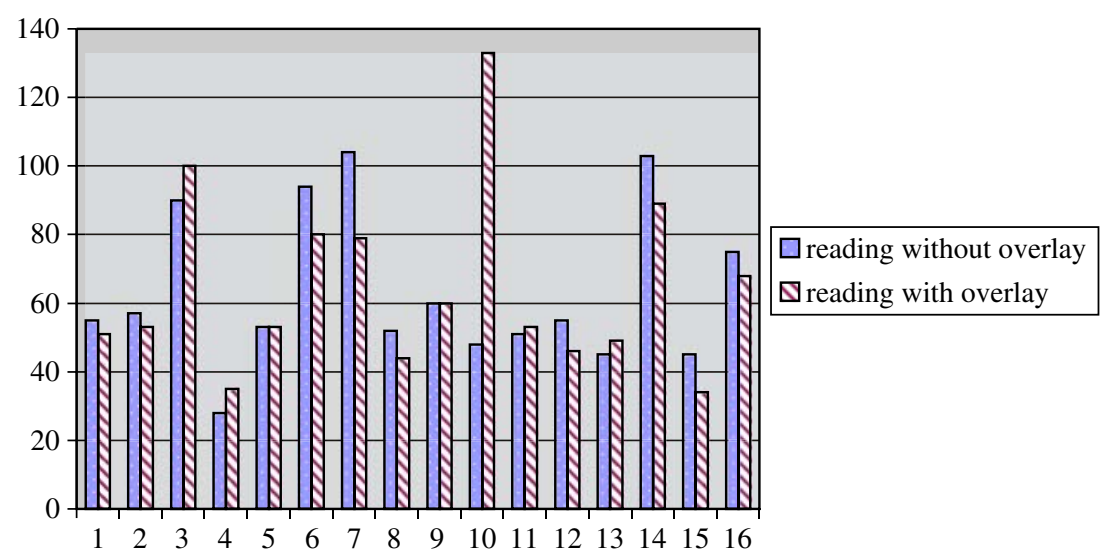

Fig. 3. Words read per minute with and without colour overlays for control children.

larger and the range of improvement greater than that seen in the control group.

Although research has shown that children who are poor or slow readers are more likely to improve in reading performance when using overlays than children without reading difficulties, this tendency is weak (Wilkins et al., 2001). The children with autism in the present sample did not show poor reading skills when reading without a colour overlay. There was no significant difference between their initial reading scores and those of their age and verbal IQ matched controls. This showed that not only were the groups matched well for reading ability but that reading scores without an overlay did not predict performance with an overlay. Correlations between verbal IQ and increase in reading rate with an overlay revealed that verbal IQ also did not appear to contribute to improvement with an overlay.

All the children with autism reported that the coloured overlays made the text clearer, whilst none reported a preference for the white (no overlay) text. Sixteen of the 19 children in the control group reported a preference for coloured overlays and three preferred the white text. All children who chose an overlay (both those with autism and controls) reported significantly fewer symptoms of visual stress when the overlay was used for reading. In the present study those who read more than $5 \%$ faster with the overlay also reported fewer symptoms of visual stress with the overlays. However, this failed to reach statistical significance. Wilkins et al. (2001) found a correlation between the number of symptoms of visual stress and an increase in reading speed with an overlay in his sample of normal children. Although none of the children in the present study asked for clarification on any of the visual stress questions, comprehension difficulties may have been in evidence and contributed to failure to replicate earlier findings. Verbal self-report measures may not be the best method for assessing symptoms of visual stress in autism, particularly where there is co-occurring intellectual impairment. Future studies should attempt to develop alternative methods for assessing visual stress in these children.

No single colour was chosen by the majority of children; instead there was a great dispersion of colours chosen. This is shown in Appendix A. The most popular was Mint-Green with $18 \%$ of the children (both controls and children with autism) selecting this colour. Similarly there was no difference in the choice of single or double overlays. $47 \%$ of children chose a double overlay, and this accounted for $57.8 \%$ of the children with autism and $36 \%$ of controls. This finding is in line with other studies showing wide variation in colour choice. In a largescale study of typically developing children the choice of colour overlay was widely distributed among the colours available and the most frequently chosen colours (rose and aqua) were chosen by less than $10 \%$ in the sample (Wilkins et al., 2001).

Despite wide dispersion in colour choices across and within groups, individuals show reliability and consistency in their colour choice across sessions. For example, the previously cited study by Wilkins et al. (2001) found that $47 \%$ of typically developing children chose exactly the same colour on two separate testing sessions and $21 \%$ chose an overlay of similar colour (neighbouring chromaticity). It was also found that children who chose exactly the same colour on both occasions showed the greatest 
improvement in reading (Wilkins et al., 2001). In the current study the improvement in reading in the group with autism also showed that the children's choice of colour was effective. Research should now be carried out to compare reading ability with a chosen overlay in comparison to other colour overlays using an autistic sample. This research will provide more evidence that benefits from these overlays are not simply due to novelty effects but it may also link particular subgroups, for example very poor readers, to certain colour choices.

The results from the Ishihara and City University colour tests showed that children who benefit from colour overlays do not have anomalous colour vision. This finding is in line with previous studies suggesting that the prevalence of colour vision anomaly in individuals with Meares-Irlen syndrome is similar to that in the general population (Evans et al., 1996a, b).

Although the mechanisms of benefit are currently uncertain, Wilkins (2003) has proposed that the mechanisms are cortical in origin. Wilkins proposes that visual distortions occur as a result of a spread of activation within the cortex that causes the cells to fire inappropriately coloured filters change the distribution of firing within the cortex and selfselected colours may alter the distribution selectively so as to reduce the excitation in hyperexcitable regions. This theory is given support by the fact that migraine and epilepsy sufferers are two groups who benefit from the use of colour overlays (Aurora \& Welch, 1998; Huang et al., 2003, 2004; Maclachlan et al., 1993).

Epilepsy is common in autism, especially in those with co-occurring intellectual impairment (Corbett, 1982; Deykin \& MacMahon, 1979; Gillberg \& Steffenburg, 1987). However individuals with normal intelligence also suffer from epilepsy (Olsson, Steffenburg, \& Gillberg, 1988; Rutter, 1970). Because there appears to be a relationship between epilepsy and autism, it was predicted that children with autism would benefit from overlays. Future research needs to address the question of whether the children with autism who benefit most from the use of colour overlays are over-represented in groups that suffer from either epilepsy and/or migraine.

Although this study provides evidence that reading performance in children with autism can be improved with use of colour overlays, these findings should be considered preliminary. For example, an important outstanding question is how the children most likely to benefit from overlays will be identified. Although findings from the present study suggest that neither reading speed without an overlay or verbal IQ predict performance with an overlay, it may be that these factors will be important when children are tested for longer periods with more complicated tasks. This is especially likely if the beneficial effects of overlays occur because they reduce cognitive demands. Longitudinal studies would provide important insights into long-term benefits of overlays and would also enable therapists and teachers to identify individuals with autism who gain most benefit.

This is the first empirical study to show that reading skills in autism are improved by the use of colour overlays. A goal for future research is to develop methods by which visual cognition in nonreading low functioning children with autism can be tested using coloured overlays. Such tests have been designed and are currently under evaluation.

\section{ACKNOWLEDGMENTS}

We would like to thank our participants and their parents and teachers for their co-operation in our research.

\section{APPENDIX}

APPENDIX A: The Colour of the Overlays Chosen by the Children with Autism and their Controls

\begin{tabular}{lcc}
\hline Colour overlay & Autism group & Control group \\
\hline No overlay & 0 & 3 \\
Rose & 0 & 1 \\
Purple & 2 & 0 \\
Mint & 4 & 3 \\
Lime & 1 & 0 \\
Blue & 0 & 3 \\
Aqua & 1 & 2 \\
Yellow + Yellow & 0 & 2 \\
Yellow + Orange & 1 & 0 \\
Orange + Orange & 1 & 1 \\
Pink + Purple & 1 & 1 \\
Pink + Rose & 1 & 0 \\
Lime + Mint & 2 & 1 \\
Mint + Mint & 2 & 0 \\
Mint + Aqua & 2 & 2 \\
Blue + Aqua & 1 & 0 \\
\hline
\end{tabular}


Coloured Overlays, Reading Ability, and Autism

\section{REFERENCES}

Aurora, S. K., \& Welch, K. M. (1998). Brain excitability in migraine: Evidence from transcranial magnetic stimulation studies. Current Opinion in Neurology, 113, 205-209.

Bartak, L., \& Rutter, M. (1975). Language and cognition in autistic children and dysphasic children. In N. O'Connor (Ed.), Cognitive Deficits and Retardation. London: Butterworths.

Bouldoukian, J., Wilkins, A. J., \& Evans, B. J. W. (2002). Randomised control trial of the effect of coloured overlays on the rate of reading of people with specific learning difficulties. Ophthalmic and Physiological Optics, 221, 55-60.

British Psychological Society. (1999). Dyslexia, literacy and psychological assessment. In R. Reason (Ed.), Report of Working Party of the Division of Educational Child Psychology. Leicester, UK: British Psychological Study.

Bryson, S. E., Clark, B. S., \& Smith, I. M. (1988). First report of a Canadian epidemiological study of autistic syndromes. Journal of Child Psychology and Psychiatry, 29, 433-441.

Chronicle, E. P., \& Wilkins, A. J. (1991). Colour and visual discomfort in migraineurs. Lancet, 338(8771), 890.

Chronicle, E. P., Wilkins, A. J., \& Coleston, D. M. (1995). Thresholds for detection of a target against a background grating suggest visual dysfunction in migraine with aura but not migraine without aura. Cephalalgia, 15, 117-122.

Cialdella, P., \& Mamelle, N. (1989). An epidemiological study of infantile autism in a French department (Rhone): A research note. Journal of Child Psychology and Psychiatry, 30, 165-175.

Corbett, J. (1982). Epilepsy and the electroencephalogram in early childhood psychoses. In J. K. Wing, \& L. Wind (Eds.), Handbook of Psychiatry. (pp. 198-202). 3London: Cambridge University Press.

Crobrinik, L. (1974). Unusual reading ability in severely disturbed children. Journal of Autism and Child Schizophrenia, 4, 163-175.

Deykin, E. Y., \& MacMahon, G. (1979). The incidence of seizures among children with autistic symptoms. American Journal of Psychiatry, 136, 1310-1312.

Diagnostic and Statistical Manual of Mental Disorders (1994) (4th ed.). Washington, DC: American Psychiatric Association.

Dunn, LI. M, Dunn, L. M., Whetton, C., \& Burley J. (1997). The British Picture Vocabulary Scale (2nd ed.). Windsor, Berks: NFER-Nelson.

Evans, B. J. W. (2001). Dyslexia and Vision. London: Whurr Publications.

Evans, B. J. W., \& Drasdo, N. (1991). Tinted lenses and related therapies for learning disabilities: A review. Ophthalmic and Physiological Optics, 11, 206-217.

Evans, B. J. W., \& Joseph, F. (2002). The effect of coloured filters on the rate of reading in an adult population. Ophthalmic and Physiological Optics, 22(6), 535-545.

Evans, B. J. W., Wilkins, A. J., Brown, J., Busby, A., Wingfield, A., Jeanes, R., \& Bald, J. (1996a). A preliminary investigation into atetiology of Meares-Irlen Syndrome. Ophthalmic and Physiological Optics, 164, 286-296.

Evans, B. J. W., Wilkins, A. J., Busby, A., \& Jeanes, R. (1996b). Optometric characteristics of children with reading difficulties who report a benefit from coloured filters. In D. Garden (Ed.), John Dalton's Colour Vision Legacy. (pp. 709-715). London: Taylor and Francis.

Fletcher, R. (1998). The City University Colour Vision Test (3rd ed.). Windsor: Keeler.

Gillberg, C. (1991). The treatment of epilepsy in autism. Journal of Autism and Developmental Disorders, 21(1), 61-77.

Gillberg, C., \& Steffenburg, S. (1987). Outcome and prognostic factors in infantile autism and similar conditions. A population-based study of 46 cases followed through puberty. Journal of Autism and Developmental Disorders, 17, 271-285.
Howlin, P. (1996). A Visit to the Light and Sound Therapy Centre. Pamplet. London: National Autistic Society.

Huang, J., Cooper, T. G., Satana, D., Kaufman, D. I., \& Cao, Y. (2003). Visual distortion associated with hyper visual neuronal activity in migraine. Headache, 43(6), 664-671.

Huang, J., Wilkins, A.J., \& Cao, Y. (2004). International Society for Magnetic Resonance in Medicine Proceedings.

Irlen, H. (1991). Reading Through Colours: Overcoming Dyslexia and Other Reading Disabilities Through the Irlen Method. New York: Avery.

Ishihara, S. (1970). Tests for Colour-Blindness. Tokyo: Kanahara Shuppan, 38 plates.

Jackowski, M. M., Strurr, J. F., Taub, H. A., \& Turk, M. A. (1996). Photophobia in patients with brain injury: Uses of light-filtering lenses to enhance contrast sensitivity and reading rate. Neurorehabilitation, 6, 193-201.

Jeanes, R., Busby, A., Martin, J., Lewis, E., Stevenson, N, Pointon, D., \& Wilkins, A. J. (1997). Prolonged use of coloured overlays for classroom reading. British Journal of Psychology, 88, $531-548$.

Kientz, M.A., \& Dunn, W. (1997). Comparison of the performance of children with and without autism on the sensory profile. The American Journal of Occupational Therapy, 51, 530-537.

Kriss, I. (2002). An Investigation into the Effects of Coloured Overlays on Reading in Dyslexics and Controls. BSc Thesis. UK: Manchester Metropolitan University.

Lockyer, L., \& Rutter, M. (1969). A five to fifteen year follow up study of infantile psychosis. III. Psychological aspects. British Journal of Psychology, 69, 46-54.

Maclachlan, A., Yale, S., \& Wilkins, A. J. (1993). Open trials of precision ophthalmic tinting: 1 year follow-up of 55 patients. Ophthalmic and Physiological Optics, 13, 175-178.

Marcus, D. A., \& Soso, M. J. (1989). Migraine and stripe-induced discomfort. Archives of Neurology, 46(10), 1129-1132.

Olsson, I., Steffenburg, S., \& Gillberg, C. (1988). Epilepsy in autism and autistic-like conditions; a population-based study. Archives of Neurology, 45, 666-668.

Ornitz, E.M. (1973). Childhood autism: a review of the clinical and experimental literature. California Medicine, 118, 21-47.

Rutter, M. (1970). Autistic children. Infancy to adulthood. Seminars in Psychiatry, 2, 435-450.

Steffenburg, S., \& Gillberg, C. (1986). Autism and autistic like conditions in Swedish rural urban areas: A population study. British Journal of Psychiatry, 149, 81-87.

Stone, R. (2002). The Light Barrier. New York: St Martin's Press.

Tanoue, Y., Oda, S., \& Kawastima, K. (1988). Epidemology of infantile autism in southern Ibaraki Spain: Differences in prevalence rates in birth cohorts. Journal of Autism and Developmental Disorders, 18, 155-166.

White, B. B., \& White, M. S. (1987). Autism from the inside. Medical Hypotheses, 24, 223-299.

Wilkins, A. J. (1994). Overlays for classroom and optometric use. Ophthalmic and Physiological Optics, 14(4), 97-99.

Wilkins, A. J. (1995). Visual Stress. Oxford: Oxford University Press.

Wilkins, A. J. (2003). Reading Through Colour. Chichester: Wiley,

Wilkins, A. J., \& Lewis, E. (1999). Coloured overlays, text and texture. Perception, 28, 641-650.

Wilkins, A. J., \& Nimmo-Smith, M. I. (1987). The clarity and comfort of printed text. Ergonomics, 3012, 1705-1720.

Wilkins, A. J., Evans, B. J. W., Brown, J. A., Busby, A. E., Wingfield, A. E., Jeanes, R. J., \& Bald, J. (1994). Doublemasked placebo-controlled trial of precision spectral filters in children who use coloured overlays. Ophthalmic and Physiological Optics, 14(4), 365-370.

Wilkins, A. J., Jeanes, R. J., Pumfrey, P. D., \& Laskier, M. (1996). Rate of Reading Test: Its reliability and its validity in the 


\section{Ludlow, Wilkins, and Heaton}

assessment of the effects of the coloured overlay. Ophthalmic and Physiological Optics, 16, 491-497.

Wilkins, A. J., Lewis, E., Smith, F., \& Rowland, E. (2001). Coloured overlays and their benefits for reading. Journal of Research Reading, 181, 10-23.

Wilkins, A. J., Nimmo-Smith, M. I., Tait, A., McManus, C., Della Sala, S., Tilley, A., Arnold, K., Barrie, M., \& Scott, S. (1984). A neurological basis for visual discomfort. Brain, 107, 989-1017.

Wilkins, A. J., Patel, R., Adjamian, R., \& Evans, B. J. W. (2002). Tinted spectacles and visually sensitive migraine. Cephalalgia, 22(9), 711-719.
Williams, D. (1999). Like Colour to the Blind. London: Jessica Kingsley Publishers Ltd.

Wing, L., \& Gould, J. (1979). Severe impairments of social interaction and associated abnormalities in children: Epidemiology and classification. Journal of Autism and Developmental Disorders, 9, 11-29.

Xiao, Y., Wang, Y., \& Fellerman, D. J. (2003). A spatially organised representation of colour in macaque cortical area V2. Nature, 421(6922), 535-539. 